Journal of Engineering and Applied Sciences 14 (Special Issue 5): 9193-9197, 2019

ISSN: 1816-949X

(C) Medwell Journals, 2019

\title{
TRIZ Applications in the Design of a Vacuum Sealed Package Opener
}

\author{
Poh Kiat Ng, K. Ganesh Kuma, Kian Siong Jee and Zi Yang Hooi \\ Faculty of Engineering and Technology, Multimedia University, \\ Jalan Ayer Keroh Lama, Bukit Beruang, 75450 Melaka, Malaysia \\ pkng@mmu.edu.my
}

\begin{abstract}
The protection, constancy, cleanliness and nutrition of products can be ensured through preservation methods. In association to this, vacuum sealed packaging has been proven to be an efficient preservation method. Nonetheless, there have been problems on the difficulty in opening these vacuum sealed packages. These problems include the frustrations faced by users (otherwise known as wrap rage) and injuries sustained due to cuts from tools or overexertion with bare hands while attempting to open these tightly sealed packages. One of the solutions to these problems lie in the design of an appropriate pocket-sized vacuum sealed pack opener that is safe to use for users of all ages. Therefore, the aim of this study is to innovate and design a vacuum sealed package opener that is not harmful to users and effectively allows users to open the packages at the same time. For this study, TRIZ tools were used. Several function models were investigated along with the cause and effect chain analysis, upon which 3 major engineering contradictions were established. These contradictions were resolved with the recommendation of 5 inventive principles, namely dynamicity, spheroidality, asymmetry, taking out and intermediary. Finally, researchers proposed a vacuum sealed pack opener equipped with a cutting wheel inserted in the upper body. This cutting wheel would cut against the sealed package's edge horizontally across and against a "V"-cavity fixed on the lower body. The new design's impact reflects on the improvement on several characteristics associated with human limitation, safety and health.
\end{abstract}

Key words: TRIZ, design innovation, vacuum packaging, vacuum seal, multifunctional, spheroidality

\section{INTRODUCTION}

Food production and supply does not always tally with the customer demand. In some places, there is a surplus production of food products whereas in some other places there is inadequate supply. Foods like juicy fruits, vegetables, mangoes, nuts, meat and many more gets spoiled quickly. It is therefore, important to improve and expand technology for preservation of food (Soccol et al., 2005). Preservation techniques warrant the security and constancy of products, so that, they present sufficient nutritional properties (Soccol et al., 2005; Leistner, 1992).

One of the best way to preserve food is by using vacuum sealed packaging. The main concept of this method is by removing the air from the package before sealing the package. By removing oxygen from the packaging, the growth of aerobic bacteria or fungi would not be possible and the amount of spoilage due to oxidation will be reduced. By using this method, seasonal food can be stored for a long time, making it even possible to be available throughout the year. This eventually helps people bring a variety of food into their diet, thereby decreasing nutritional inadequacies. Researchers also, contend that while vacuum packaging and refrigeration can go hand-in-hand as an effective technique to preserve germination capacity, it was found that vacuum packaging by itself was more than enough (Croft et al., 2012).

The vacuum sealed package is usually sealed tightly using polyvinylidene chloride and ethylene vinyl alcohol vacuum packs. These material have aremarkable barrier against water and oxygen, thus having superior chemical resistance toalkali and acid. They are also, insoluble in oil and organic solvents and impervious to mold, bacteria and insects. Sometimes, they are even manufactured with multi-layer structures to have a strong oxygen-barrier in order to prevent the penetration of oxygen and bacteria into the food. This results in the thickness of the vacuum sealed package to be as large as $2 \mathrm{~mm}$. With this thickness, it is going to be difficult to unseal the package without a proper tool due to the strength of the seal. Usually, people would use sharp tools such as scissors and blades to open the seal. However, these methods may cause injury to them. Hence, the problem statement is as such: problem statement; It is difficult to open a vacuum sealed package.

Corresponding Author: Poh Kiat Ng, Faculty of Engineering and Technology, Multimedia University, Jalan Ayer Keroh Lama, Bukit Beruang, 75450 Melaka, Malaysia, pkng@mmu.edu.my 
Literature review: Most market outlets retail products in vacuum sealed packages. Even though this sort of packaging is robust, easily set in a display, prevents shoplifting and permits customers to view products with ease. However, it appears that there are numerous issues with the vacuum sealed packages. These packages are difficult to open and more often than not, the techniques used to open them are harmful. A particular phenomenon called the wrap rage started to get acknowledged by the public due to the annoyance from the difficulty in opening tightly sealed packages such as vacuum sealed packages (Anonymous, 2006).

It was found that $99 \%$ of 2000 individuals who exceeded 50 years of age concurred that tightly sealed packages (such as vacuum sealed packages) have turned out to be very hard to open in the past decade (BBC News, 2004). Apart from that vacuum sealed packages have sharp edges that could cause injuries. Customers sometimes tend to use potentially unsafe apparatuses like study blades, scissors and ice-picks with the intention to slice or cut open these sealed packages (ABC News, 2006). At times, they even use their own teeth to rip open these packages, leading to a cracked tooth or even a loss of a tooth. Approximately, 60,000 individuals visit hospitals every year for major and minor treatments due to injuries from opening vacuum sealed packages (Moore, 2003). These light and serious injuries involved cuts on the fingers, cuts on the hand, wrist sprains, bruises and shoulder sprains (BBC News, 2004). Efforts to open tightly sealed vacuum packages prompted about 6500 admissions and visits to the hospitals (Wenzel, 2008).

One of the product challenges faced would be to propose a pocket-sized tool design of an appropriate vacuum sealed package opener that can address the preceding issues. Hence, this study aims to innovate and design a vacuum sealed package opener that is not harmful to users and effectively allows users to open the packages at the same time.

\section{MATERIALS AND METHODS}

The TRIZ approach was used for this study, since, it is known as an effective problem-solving tool created from the research of inventive patterns from international patent literature (Yeoh, 2014; Yeoh et al., 2015). Similar approaches used in previous studies have also proven that TRIZ tools are capable of further innovating even the most basic products such as screwdrivers and knobs (Ng and Jee, 2016; Ng et al., 2016a, b). Upon the identification of the main problem, there are 3 major TRIZ tools used in this project, namely the function analysis, cause and effect chain analysis and engineering contradiction. Upon identifying the engineering contradictions the system parameters will be identified

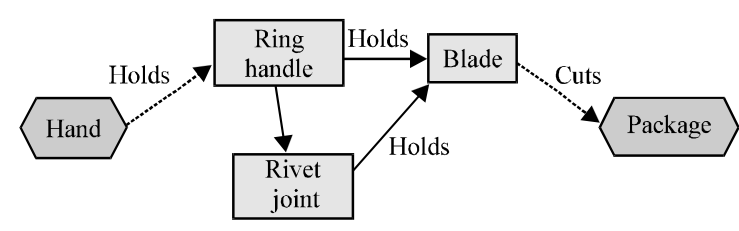

Fig. 1: Function model for a typical scissors

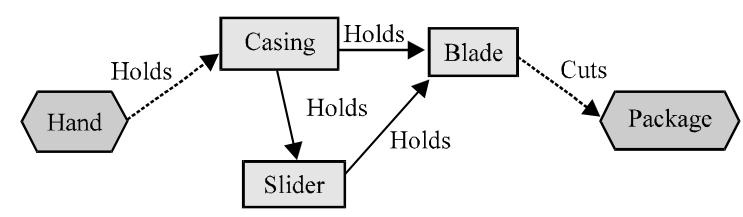

Fig. 2: Function model for a typical paper blade

and cross-referenced in the contradiction matrix in order to know the recommendations of the TRIZ inventive principles.

Function analysis: For the function analysis for both the typical scissors and study blade can be seen in Fig. 1 and 2. For the scissors, the ring handle and rivet joint holds the blades. The cutting process would then start with the hand insufficiently holding the ring handle (due to lack of contact area) and this would also, cause the blade of the scissors to insufficiently cut the vacuum sealed package.

For the study blade, the blade would be held by the slider and casing which also holds the slider at the same time. However, during the cutting process, the hand would similarly insufficiently hold the casing which would then cause the blade to insufficiently cut the package.

The outcome of the function analyses concludes that these domestic tools may not be built to cut vacuum sealed packages effectively and the major difficulties could lie in holding the tool during the cutting process and ineffectively cutting the package. Even, if these domestic tools were further modified to research on vacuum sealed packages, the increase in dimensions or the thickness of the blade would still be a risk to the users.

Cause and Effect Chain (CEC) Analysis: A Cause and Effect Chain (CEC) analysis was done in order to identify the root cause (s) behind the problem defined in the previous section. Figure 3 shows the CEC analysis conducted for the problem statement which states that it is difficult to open the vacuum sealed package injured in the process. The user can get injured on the accounts of two scenarios, namely due to the use of an inappropriate 


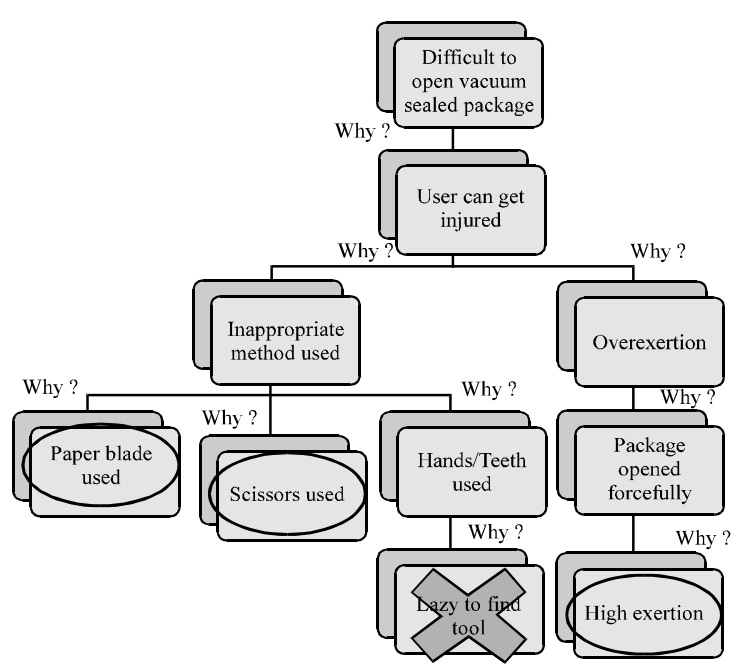

Fig. 3: Cause and effect chain analysis

method or overexertion. Over exertion happens because the package is opened forcefully (even when using a cutter) and this is because the vacuum sealed package generally, requires high exertion to be opened. On the other hand when an inappropriate method is used, it is likely due to the user utilising common tools that are used for other general means. Two of these common tools include the study blade and scissors. Another method may involve the users using their hands or teeth, mostly due to the laziness of searching for a common tool nearby (hence, this, reason is excluded). Therefore, the major root causes identified from the problem definition include the use of study blades, the use of scissors and high exertion.

Engineering contradictions, system parameters and inventive principles: From the potential root-causes identified, there were about 5 engineering contradictions developed. The system parameters were identified based on the characteristics of the improving and worsening parameters in these contradictions. The contradictions can be found in the subsequent paragraphs.

Scissors cut package, blades reciprocate: If a scissors were to be used to cut the package, then the package can be cut through without much issues (reliability) but the blades need to reciprocate regularly to cut across completely (length of moving object).

Scissors rings held, small area: If a scissors were to beused to cut the package, then the fingers can effectively fit the rings (shape) but the grip surface area while cutting would be small (area of moving object).
Paper blade used, higher energy: If a study blade were to be used, then it would be easy and straightforward to use (ease of operation) but the amount of energy exerted to completely cut the package increases (use of energy by moving object).

Forceful cut, high strength: If high exertion were to be used to cut through the package, then the cutter can successfully cut through the package (force) but the amount of strength used would be high (strength).

Faster cut, injured user: If high exertion were to be used to cut through the package, then the cutting would be faster (Speed) but the cutting tool might injure the user (object-generated harmful factors).

Based on the identified system parameters, several inventive principles from the matrix of contradiction can be recommended to aid the proposal of solutions for this project. Out of all the 40 inventive principles (Yeoh, 2014; Yeoh et al., 2015) only 5 of them were used for the proposed solution, namely dynamicity, spheroidality, intermediary, asymmetry and taking out.

\section{RESULTS AND DISCUSSION}

Spheroidalit; cutting wheels: The principle of curvature was used by introducing cutting wheels in order to lessen the amount of force used to cut the package. Since, the contact point between the blade area and package's surface area is smaller, the stress induced is higher, hence, allowing the packaging to be easily cut while reducing the probability of cutting the user. The design of the circular blade also, allows the cutting of the plastic vacuum packaging from both directions, though it would require more force as the cutting surface area is larger. It also would not dent the groove as the teethed cutting blade would though it would still create scratches from excessive punching force.

Intermediary-" $V$ " cavity: The principle of intermediary was used by introducing a "V" cavity in order to lessen the amount of energy exerted through the cutting wheel to cut through the package. The "V" cavity would intermediate between the cutter and the lower body. Since, a cutting wheel is used as the punch tip and the groove as the die, the cutting mechanism would be based on the press braking or brake forming process. This process is a deformation process which is commonly used on metal sheets where it is formed or dented along a straight axis through a punch and die set. Popular punch and die sets are usually "V" and " $U$ "-shaped or channel-shaped. 
(a)

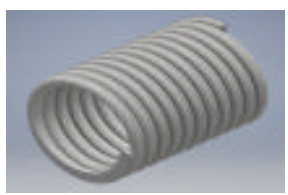

(e)

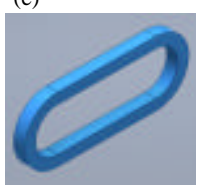

(b)

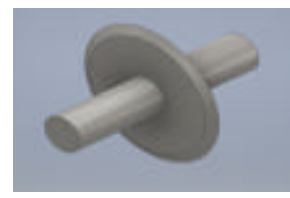

(f) (c)

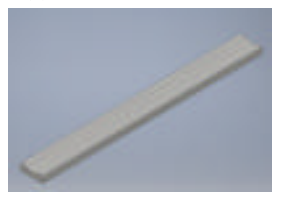

(g) (d)
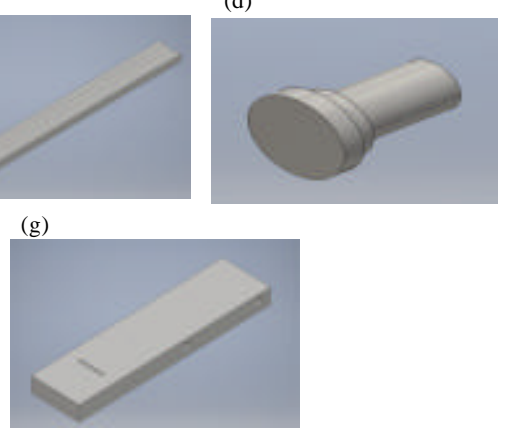

Fig. 4: Mechanical components of the vacuum sealed package opene: a) Spring; b) Cutting blade; c) Groove; d) Screws; e) Brackets; f) Upper body and g) Lower body

Dynamicity-rack and pinion concept: The principle of dynamicity was used by introducing the rack and pinion concept in order to provide a continuous rolling effect for the cutting wheel when cutting rather than a reciprocating effect by a scissors or a dragging effect by a blade. For the feeding mechanism, the rack and pinion concept would be more suitable for feeding and removing the plastic vacuum packaging from the device itself. Rotational motion applied to the pinion causes the rack to move relative to the pinion, thereby translating the rotational motion of the pinion into linear motion. Vice versa, the linear motion of the rack can be translated to the rotationa motion of the pinion while being stationary.

Asymmetry and taking out-upper and lower body (casing): Firstly, the principle of asymmetry was used by introducing two parts (upper and lower body) with large surface areas for the fingers to effectively grip. These two parts are asymmetrical to each other. This would resolve the issue gripping small surface areas such as the rings of a scissors. Besides that the principle of taking out was also, used through upper body because it also acts as a casing for the cutting wheel. This situation involves relocating the cutting wheel into the casing to avoid it from being exposed to the user. Hence, no matter how fast or slow the cutting process is executed, the user would not be cut by the cutting blade as it is separated from the user through the casing.

Final product: From the proposed mechanisms, the vacuum sealed package opener design is created and it is made from the helical compression (Fig. 4).

The components are assembled as shown in Fig. 5 and 6 . The spring is inserted into the circular hole of the upper body. It acts as a support for the whole device. The cutting wheel is inserted into the square hole of the upper body. It acts as a cutter to cut or create a tear line or

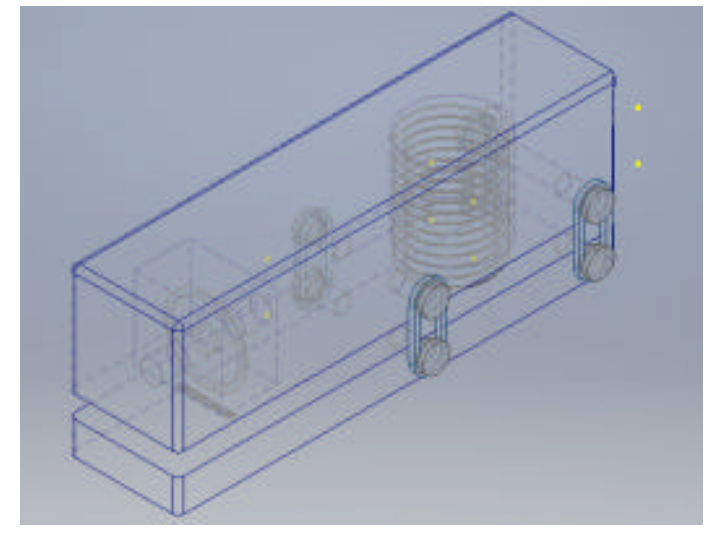

Fig. 5: Whole assembly of vacuum sealed package opener

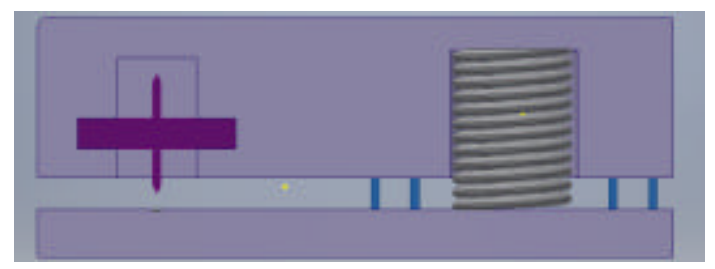

Fig. 6: Cross-sectional view of the vacuum sealed packaging opener

opening line for the vacuum sealed package. The wheel only rotates in a single axis. The groove is fixed on the lower body. Besides that a total of 8 screws will be used to thighten the brackets and the body of the opener. The brackets allow the motion of the upper body to be parallel to lower body of the opener. Operational procedures for using the vacuum sealed package opener:

- Insert the vacuum sealed package into the feeder of the opener 
- Press on the opener and slide it along the vacuum sealed package

- The vacuum sealed package would be cut across completely, if the plastic is thin

- If the plastic is thick, it would create a tear line on the plastic packaging

- After creating the tear line or completely cutting across the plastic package, release the device

- Remove the package from the device

- If there is a tear line from the above process, tear along the tear line (with minimal exertion) to completely open the vacuum sealed package

\section{CONCLUSION}

The pocket sized vacuum sealed package opener with cutting blade technology and safety features significantly eases the process of unsealing vacuum packages. It isa product designed with a safe body that covers the blade, hence, avoiding any injury to user. It is also, user friendly and requires minimal force to be operated, allowing it to be used by all ages (from small children to the elderly population). The vacuum sealed package opener is also estimated to be a low cost product solution for day-to-day problems and is estimated to cost much $<\mathrm{US} \$ 25$ based on the small and simple parts involved. Eventually, this new product can significantly reduce domestic household injuries and improve human performance.

\section{REFERENCES}

ABC News, 2006. Wrap rage. ABC News, Australia. http://abcnews.go.com/Business/Video/player Index?id=2692861

Anonymous, 2006. Consumer reports: The oyster awards. Consumers Union of US Inc, San Francisco, California. https://web.archive.org/web/ 20070613044108/http:/www.consumerreports.org/cr o/personal-finance/hardtoopen-packages306/overview/index.htm
BBC News, 2004. Wrap rage hitting the over-50s. BBC News, London, UK. http://news.bbc.co.uk/2/hi /business/3456645.stm

Croft, M., A. Bicksler, J. Manson and R. Burnette, 2012. Vacuum sealing vs. refrigeration: Which is the most effective way to store seeds? ECHO. Asia Notes, 14: $1-6$.

Leistner, L., 1992. Food preservation by combined methods. Food Res. Intl., 25: 151-158.

Moore, J., 2003. City diary: Slice of life in the kitchen. Telegraph Media Group Limited, UK. http://www.telegraph.co.uk/finance/comment/citydi ary/2868234/City-diary.html

Ng, P.K. and K.S. Jee, 2016. Design and development of an ergonomic milling machine control knob using TRIZ principles. Am. J. Appl. Sci., 13: 451-458.

Ng, P.K., K.S. Jee and B.S. Choong, 2016b. Design innovation of a manual screwdriver using the inventive principles of TRIZ. Middle East J. Sci. Res., 24: $372-378$.

Ng, P.K., K.S. Jee and B.S. Choong, 2016a. Designing an ergonomic inward directional screwdriver using TRIZ inventive principles. Proceedings of the International Conference on Applied and Engineering Physics, February 26-27, 2016, Multimedia University, Penang, Malaysia, pp: 1-10.

Soccol, M.C.H., M. Oetterer, C.R. Gallo, M.H.F. Spoto and D.O. Biato, 2005. Effects of modified atmosphere and vacuum on the shelf life of Tilapia: Oreochromis niloticus fillets. Braz. J. Food Technol., 8: 7-15.

Wenzel, E., 2008. Killing the oyster pack. CBS Interactive Company, San Francisco, California, USA. https://www.cnet.com/news/uber-ceo-travis kalanick-isnt-leaving-but-hes-going-to-changehuffington-coo/

Yeoh, T.S., 2014. TRIZ: Systematic innovation in business and management. Firstfruits Sdn Bhd, Selangor, Malaysia.

Yeoh, T.S., T.J. Yeoh and C.L. Song, 2015. TRIZ: Systematic innovation in manufacturing. Firstfruits Sdn Bhd, Petaling Jaya, Malaysia. 